den im Vordergrund stehenden gesetzgeberischen Motiven möchte der Bundesgesetzgeber einerseits mit $\$ 7$ Abs. 2 S. 1 UmwRG der normstrukturellen Vergleichbarkeit von SUP-pflichtigen Plänen und Programmen i.S.d. $\$ 1$ Abs. 1 S. 1 Nr. 4 UmwRG mit den gem. \47 VwGO i. V.m. dem Landesrecht der prinzipalen Kontrolle durch die Oberverwaltungsgerichte zugeordneten Handlungsformen der Satzung und der Rechtsverordnung Rechnung tragen. Andererseits zielen die mit dem Investitionsbeschleunigungsgesetz 2020 eingefügten $₫ 48$ Abs. 1 S. 1 Nr. 3a VwGO und $\$ 48$ Abs. 1 S. 1 Nr. 3b VwGO auf die Beschleunigung der gerichtlichen Kontrolle von Infrastrukturvorhaben mit Bedeutung für die Energiewende (Windenergieanlagen an Land mit einer Gesamthöhe von über 50 Meter, KWK-Anlagen ab einer Feuerungswärmeleistung von 50 Megawatt) durch Beschränkung auf eine Tatsacheninstanz. Infolge der Neuregelungen ist bei der Bestimmung der gerichtlichen Zuständigkeit und des Instanzenzugs im Immissionsschutzrecht deutlich stärker als bisher zu differenzieren. Während es grundsätzlich beim dreistufigen Instanzenzug mit Verwaltungsgericht, Oberverwaltungsgericht und Bundesverwaltungsgericht bleibt, gilt für einen signifikanten Teil immissionsschutzrechtlicher Streitigkeiten nunmehr ein zweistufiger Instanzenzug mit dem Oberverwaltungsgericht als Eingangsinstanz und dem Bundesverwaltungsgericht als Revisionsinstanz. Zudem verankert das Investitionsbeschleunigungsgesetz 2020 in $\$ 63 \mathrm{BImSchG}$ das Entfallen der aufschiebenden Wirkung für Rechtsbehelfe Dritter gegen Windenergieanlagen an Land mit einer Gesamthöhe von über 50 Meter.

Open Access. Dieser Artikel wird unter der Creative Commons Namensnennung 4.0 International Lizenz veröffentlicht, welche die Nutzung, Vervielfältigung, Bearbeitung, Verbreitung und Wiedergabe in jeglichem Medium und Format erlaubt, sofern Sie den/die ursprünglichen Autor(en) und die Quelle ordnungsgemäß nennen, einen Link zur Creative Commons Lizenz beifügen und angeben, ob Änderungen vorgenommen wurden.

Die in diesem Artikel enthaltenen Bilder und sonstiges Drittmaterial unterliegen ebenfalls der genannten Creative Commons Lizenz, sofern sich aus der Abbildungslegende nichts anderes ergibt. Sofern das betreffende Material nicht unter der genannten Creative Commons Lizenz steht und die betreffende Handlung nicht nach gesetzlichen Vorschriften erlaubt ist, ist für die oben aufgeführten Weiterverwendungen des Materials die Einwilligung des jeweiligen Rechteinhabers einzuholen.

Weitere Details zur Lizenz entnehmen Sie bitte der Lizenzinformation auf http://creativecommons.org/licenses/by/4.0/deed.de.

Open Access funding enabled and organized by Projekt DEAL.

\title{
Der vernünftige Grund des Tierschutzgesetzes und die Tötung von Tieren in Zoos - ein unerkannter Widerspruch? Christian Arleth und Nikklas-Jens Biller-Bomhardt
}

Zoos verstehen sich nicht nur als Stätten der Arterhaltung. Sie sind nach den zwingenden Genehmigungsvoraussetzungen des $\int 42$ Abs. 3 Bundesnaturschutzgesetz (BNatSchG) unter anderem auch verpflichtet, den biologischen Bedürfnissen bei der Haltung der Tiere (Nr. 1), der Pflege der gefangenen ${ }^{1}$ Individuen nach dem guten Stand veterinärmedizinischer Praxis (Nr. 2) sowie der Einhaltung der Vorschriften des Tierschutz- und des Artenschutzrechts (Nr. 4) Rechnung zu tragen. Umso erstaunlicher ist es, dass in Zoos Tötungen von dort lebenden Tieren ihre Ursache häufig in monetären oder zuchtgenetischen Beweggründen haben. Mit dem folgenden Beitrag soll die besondere rechtliche Stellung von Zoos im Hinblick auf den im deutschen Tierschutzrecht verankerten Lebensschutz jedes einzelnen Tieres und die sich daraus ergebenden Implikationen für die Prüfung des vernünftigen Grunds der SS 1 S. 2, 17 Nr. 1 Tierschutzgesetz (TierSch $G)$ anhand verschiedener Fallbeispiele aufgezeigt werden.

\section{Einführung}

Das mediale Echo war immens, als der Zoo Neumünster zu Beginn der Covid-19-Pandemie im April 2020 verkün-

\section{Dr. Christian Arleth,}

Juristischer Referent der Stabsstelle des Berliner

Landestierschutzbeauftragten, Senatsverwaltung für Justiz,

Verbraucherschutz und Antidiskriminierung,

Berlin, Deutschland

Nikklas-Jens Biller-Bomhardt,

Richter in einer Kammer für Bausachen,

Landgericht Frankfurt am Main,

Frankfurt am Main, Deutschland dete, dass ein Notfallschlachtplan für die in diesem Zoo lebenden Tiere aufgrund der fehlenden Besucher und damit einhergehender fehlender Einnahmen für die Tierhaltung erarbeitet wurde, um festzulegen, welche Tiere als erstes getötet werden müssten. Auch wenn besonders dieser Notfallplan aus Neumünster Aufmerksamkeit erfuhr, ist er kein Einzelfall, sondern wurde von der Zooleitung lediglich vergleichsweise offen kommuniziert. ${ }^{2}$ Ebenfalls breite Aufmerksamkeit erfuhr die Anordnung des Zoomanagements in Nürnberg im Jahre 2015 zur Tötung von insgesamt 60 dort lebenden Tieren. ${ }^{3}$ Die Tötungen wurden für 33 der Tiere mit Verfütterung der Getöteten an andere Zootiere begründet, für die übrigen 27 damit, dass der Platz im Zoo für diese nicht ausreiche und daher nur diejenigen dersel-

1) Von „Gefangenschaft“ spricht der Gesetzgeber im Bundesnaturschutzgesetz im Hinblick auf die Haltung von Tieren in Zoos selbst, vgl. $\$ 42$ Abs. 3 Nr. 7 lit. b) BNatSchG.

2) Vgl. exemplarisch die Berichte bei Redaktionsnetzwerk Deutschland v. 13.4.2020, Stand 2.3.2021, abrufbar unter https://www. rnd.de/panorama/corona-krise-tierpark-fuhrt-liste-von-tierendie-getotet-werden-mussten-SGLY2BYZRFB4HDSWGQW7G3RN74.html, in der Süddeutschen Zeitung v. 15.4.2020, Stand 2.3.2021, abrufbar unter https://www.sueddeutsche.de/ panorama/corona-krise-zoo-notschlachtungen-1.4877112, und dem Spiegel v. 15.4.2020, Stand 2.3.2021, abrufbar unter https://www.spiegel.de/panorama/coronakrise-zoo-in-neumuenster-erstellt-notschlachtplan-fuer-tiere-a-e045797c-b868-4780932d-c1c1d2c19105.

3) 14 Hirsche, 10 Ziegen, 7 Schafe, 14 Antilopen, 4 Steinböcke, 7 Rinder, 2 Pferde, 1 Esel und 1 Büffel. 
ben Art weiterleben dürften, die für die Erhaltung eines möglichst variablen Genpools zur weiteren Zucht besonders wertvoll seien. ${ }^{4}$ Derselbe Zoo hatte bereits 2011 einen Pavian, der in der Rangordnung aufsteigen wollte, ohne erkennbare Bemühungen einer Verlegung in einen anderen Zoo getötet. Erst im Herbst 2020 machte der Zoo wieder von sich reden, nachdem der Zoodirektor die Prüfung der Tötung des 14-jährigen asiatischen Löwen Subali wegen mutmaßlicher Zeugungsunfähigkeit öffentlich gemacht hatte. ${ }^{5}$ Dabei lehnte er sogar die von einem Radiosender nach massivem öffentlichen Protest organisierte und finanzierte Abgabe Subalis in eine Auffangstation in Südafrika, die auf Löwen aus Zoos spezialisiert ist, als ,,weder realisierbar noch notwendig" ${ }^{6} \mathrm{ab}$. Auch der Tierschutzgesetzkommentator Ernst Metzger befasst sich in einem aktuellen Beitrag in dieser Zeitschrift ${ }^{7}$ mit der Thematik und bespricht hierbei zugleich den 2019 in der Zeitschrift für evangelische Ethik (ZEE) erschienenen Beitrag des evangelischen Theologen Clemens Wustmans und des Nürnberger Zoodirektors Dag Encke ${ }^{8}$. Nach hier vertretener Ansicht bleibt Metzger dabei jedoch zentrale neuere Aspekte der juristischen Diskussion schuldig wie etwa die Implikationen der grundlegenden Entscheidung des BVerwG zum Töten männlicher Küken vom 13.6.2019 und das damit höchstrichterlich bestätigte tierschutzrechtliche Lebensschutzprinzip sowie die Gewichtung entgegengesetzter wirtschaftlicher Interessen auch für die vorliegende Thematik (dazu unter 4.1). Auch sein vages Résumé (,Einiges bleibt offen.“"), in dem er unspezifisch konstatiert, man werde ,den Zoos Gestaltungsbefugnisse oder Beurteilungsspielräume einzuräumen haben", ist äußerst kritikwürdig, leistet es doch Rechtsunsicherheit, Uneinheitlichkeit und einem noch größeren Vollzugsdefizit im Tierschutzrecht ${ }^{10}$ Vorschub. Hinzu kommt, dass Betrachtungen wie die der Neumünsteraner oder Nürnberger Zoos, die immer auch wirtschaftliche Motive haben (im Beispielsfall des Neumünsteraner Zoos sogar in erster Linie), diametral dem Verhältnis vieler Menschen der heutigen Gesellschaft zu (anderen) Tieren entgegenstehen. Menschen betrachten sie als Familienmitglieder, mit denen sie das eigene Leben teilen, stellen ihre Reduzierung und Objektifizierung zu sogenannten ,Nutztieren“ zunehmend in Frage und lehnen ihre teilweise immer noch praktizierte artwidrige Dressur in Zoos und Zirkussen im Rahmen von Unterhaltungsshows (nicht selten mit auch für Menschen gefährlichen Unfällen) zunehmend $a b .{ }^{11}$ Die tiefe Anteilnahme vieler Personen für die verstorbenen Menschenaffen, als das Affenhaus im Krefelder Zoo zu Beginn des Jahres 2020 abbrannte, verwundert daher nicht. ${ }^{12}$ Das sich wandelnde Mensch-Tier-Verhältnis drückt sich auch in der in Deutschland kontinuierlich steigenden Anzahl an Menschen aus, die eine am Tierwohl orientierte Ernährung praktizieren. So ernähren sich ca. 1. Mio. Menschen vegan $^{13}$ und ca. 6 . Mio. Menschen ${ }^{14}$ vegetarisch. Die veränderte Wahrnehmung des Tieres und seiner Bedeutung durch die Gesellschaft tangiert auch die Gesetzgebung und Rechtsprechung. So hat z.B. der Bundestag mit verfassungsändernder Zwei-Drittel-Mehrheit zum 1.8.2002 den Tierschutz in Verfassungsrang erhoben und in Art. 20a GG zum Staatsziel erklärt. In der Gesetzesbegründung zur Verfassungsänderung heißt es: „Ethischem Tierschutz wird heute ein hoher Stellenwert beigemessen. Entscheidungen verschiedener Gerichte lassen die Tendenz in der Rechtsprechung erkennen, diesem Bewusstseinswandel bei der Verfassungsauslegung Rechnung $z u$ tragen. Die Rechtsprechung kann dies aber angemessen nur vollziehen, wenn der Gesetzgeber den Tierschutz ausdrücklich in das Gefüge des Grundgesetzes einbezieht." 15 Diese veränderte Wahrnehmung zeichnet sich auch in einfachgesetzlichen Regelungen $\mathrm{ab}$. So ordnet z.B. die Vorschrift des $\$ 811 \mathrm{c}$ Abs. 1 ZPO an, dass Tiere grundsätzlich als unpfändbar gelten, wenn diese im häuslichen Bereich mit dem Vollstreckungsschuldner leben und nicht zu Erwerbszecken gehalten werden. Anders als die übrigen Pfändungsverbote des $₫ 811 \mathrm{ZPO}$ ist der Telos des $\$ 811 \mathrm{c}$ ZPO zuvorderst die Beachtung der emotionalen Beziehung des Schuldners zu dem nicht menschlichem Tier. ${ }^{16}$ Vor diesem Hintergrund erscheint es angebracht, die Praxis der deutschen Zoos die Tötung im eigenen Zoo gehaltener Tiere aufgrund wirtschaftlicher ${ }^{17}$, zucht-genetischer ${ }^{18}$, gesundheitlicher sowie anderer Gründe - einer kritischen rechtlichen Würdigung zu unterziehen. Zu untersuchen ist, ob das Töten von Zootieren aus diesen Gründen als von einem ,vernünftigen Grund“ im Sinne der SS 1 S. 2, 17 Nr. 1 TierSchG gedeckt angesehen werden kann. Obwohl die Schadenszufügungs- und Tötungsverbote der zitierten Vorschriften als Ausdrücke ethisch motivierten Tierschutzes bereits einfachgesetzlich für sich stehen und multipolar gelten, da sie nicht zwischen staatlichen, teilstaatlichen und rein privaten Adressaten unterscheiden, scheint jedenfalls im Hinblick

4) Vgl. die Berichterstattung über dieses Vorgehen bei PETA, Stand 2.3.2021, abrufbar unter https://www.peta.de/zoo-nuernberg-wiederholtes-toeten-gesunder-tiere-ohne-vernuenftigen-grund-peta und bei Nordbayern, Stand 2.3.2021, abrufbar unter https://www.nordbayern.de/region/nuernberg/im-tiergarten-nurnberg-mussten-60-tiere-getotet-werden-1.4987329.

5) Podcast "Horch amol“ der Nürnberger Nachrichten v. 28.10.2020, Stand 2.3.2021, abrufbar unter https://www.nordbayern.de/region/nuernberg/horch-amol-tiere-toten-im-nurnberger-tiergarten-1.10559372.

6) Nordbayern.de v. 25.11.2020, „Chaos um Löwe Subali geht weiter: Jetzt spricht Tiergarten-Chef", Stand 2.3.2021, abrufbar unter https://www.nordbayern.de/region/nuernberg/chaosum-lowe-subali-geht-weiter-jetzt-spricht-tiergarten-chef-1. 10633225.

7) Metzger, NuR 2020, $821 \mathrm{ff}$.

8) Wustmans/Encke, ZEE 2019 (Heft 4), $250 \mathrm{ff}$.

9) Metzger, NuR 2020, 821, 827.

10) Weiterführend dazu Bülte, NJW 2019, $19 \mathrm{ff}$; ders., GA 2018, $35 \mathrm{ff}$

11) Vgl. Wilkeneit/Schulz, Der Hund in der Erwerbsarbeit der Dienstleistungsgesellschaft. Eine Untersuchung der Merkmale und Bedingungen qualifizierter Tätigkeiten von Tieren am Beispiel von Hunden, in: Pfau-Effinger/Buschka (Hrsg.), Gesellschaft und Tiere: Soziologische Analysen zu einem Ambivalenten Verhältnis, 2013, S. 117, 155f.; Sebastian, Subjekt oder Objekt?: Ambivalente gesellschaftliche Mensch-Tier-Beziehungen als Resultat kultureller Aushandlungs- und Wartungsprozesse, in: Diehl/Tuider (Hrsg.), Haben Tiere Rechte?: Aspekte und Dimensionen der Mensch-Tier-Beziehung, 2019, S. 69, 73.

12) Z.B. stellten viele Trauernde Gedenkkerzen auf und legten Gedenkkarten und Andachtsblumen am Unglücksort nieder mit Aufschriften wie „Ihr Affen im Himmel, ich vermisse euch sehr“" oder „Ihr wart es wert, so sehr geliebt zu werden“, vgl. Polonyi, „Der Menschenaffe“, in: Der Spiegel, Nr. 31/2020 v. 25.7.2020, S. 63.

13) Statista/IfD Allensbach, Personen in Deutschland, die sich selbst als Veganer einordnen oder als Leute, die weitgehend auf tierische Produkte verzichten, in den Jahren 2015 bis 2019, Stand 2.3.2021, abrufbar unter de.statista.com/statistik/daten/ studie/445155/umfrage/umfrage-in-deutschland-zur-anzahlder-veganer/

14) Statista/IfD Allensbach, Anzahl der Personen in Deutschland, die sich selbst als Vegetarier einordnen oder als Leute, die weitgehend auf Fleisch verzichten, von 2014 bis 2019, Stand 2.3.2021, abrufbar unter de.statista.com/statistik/daten/studie/173636/ umfrage/lebenseinstellung-anzahl-vegetarier/.

15) BT-Drs. $14 / 8860$, S. 3.

16) BT-Drs. $11 / 5463$, S. 7; Gruber, in: Krüger/Rauscher, Münchener-Kommentar-ZPO, 5. Aufl. 2016, \$811c Rdnr. 1. Die übrigen Pfändungsverbote dienen zunächst sozialen Gründen, vgl Seiler, in: Thomas/Putzo, ZPO, 41. Aufl. 2020, \$811 Rdnr. 1.

17) Wie die geplante Tötung der Tiere im Zoo Neumünster im Rahmen der Notfallschlachtung.

18) Wie die Tötung der Tiere im Zoo Nürnberg 2015. Ähnliche Fälle hatten das AG Marburg, Urt. v. 17.6.2010 - 14 Ds $181 \mathrm{Js}$ 17116/08, BeckRS 2010, 17656 (drei sibirische Tigerbabys), und das OLG Naumburg, Beschl. v. 28.6.2011 - 2 Ss 82/11, BeckRS 2011, 25165 (Tigerbabys, die einer Paarung eines sibirischen Tigers und eines Sumatra-Tigers entstammten), zu entscheiden. 
auf Art. 20a $\mathrm{GG}^{19}$ ein genauerer Blick auf die rechtsdogmatische Begründung der Geltung in den drei aufgezeigten divergierenden Adressatenkategorien angebracht (3). Hinsichtlich des Gewährleistungsumfangs des Staatsziels ergeben sich dabei aber auch für die wenigen Zoos in rein privater Trägerschaft im Ergebnis keine Unterschiede (3.2 und 3.3). Die gängige Praxis der Tiertötungen in deutschen Zoos ist deshalb immer auch an Art. 20a GG zu messen. Hierfür ist nach kurzer Darstellung der dogmatischen Grundlagen zum vernünftigen Grund (2) und zur besonderen rechtlichen Stellung des Zoos als öffentlicher Institution mit einem Bildungsauftrag (3.1) die Staatszielbestimmungsbindung (3.2 und 3.3) zu untersuchen. Schließlich werden verschiedene Fallgruppen von Zootiertötungen dargestellt und es wird geprüft, ob diese noch vom vernünftigen Grund im Sinne der $\int \$ 1$ S. 2, 17 Nr. 1 TierSchG rechtlich gedeckt sind (4).

\section{Der „,vernünftige Grund“"im Tierschutzgesetz}

Der ,vernünftige Grund“ in $\int S 1$ S. 2, 17 Nr. 1, 18 Abs. 1 Nr. 1 und Abs. 1 TierSchG ist der zentrale unbestimmte Rechtsbegriff des deutschen Tierschutzrechts, das Einfallstor für wirtschaftliche, wissenschaftliche oder auch naturschutzrechtliche Interessen, die anhand der Umstände der konkreten Einzelfälle und anhand der beteiligten Rechtsgüter mit den Lebens- und Integritätsinteressen der Tiere abzuwägen sind. ${ }^{20}$ Die Geltung des Tierschutzrechts und damit auch des Abwägungsprinzips im Rahmen des ,vernünftigen Grundes“ für dem Naturschutzrecht unterfallende wild lebende Tierarten regelt deklaratorisch die allgemeine Vorschrift des $\$ 37$ Abs. 2 S. 1 BNatSchG und als lex specialis für in Zoos gehaltene Tiere eigentlich wild lebender Arten $\$ 42$ Abs. 3 Nr. 4 BNatSchG. Ein ,,vernünftiger Grund" für die Zufügung von Schmerzen, Leiden oder Schäden bei einem Tier im Sinne dieser Normen liegt nur dann vor, wenn das intendierte Verhalten einem schutzwürdigen menschlichen Interesse dient, das auch unter den konkreten Umständen schwerer wiegt als das Interesse am Schutz des Tieres. ${ }^{21} \mathrm{Ob}$ ein Grund als ,vernünftig“ einzustufen ist, ist für jeden Einzelfall im Rahmen einer umfassenden Abwägung aller betroffenen Interessen gesondert zu prüfen. ${ }^{22}$ Schematische Kategorisierungen von ,,anerkannten Gründen“ und die Bildung von abstrahierenden Fallgruppen werden der obligatorischen Interessenabwägung auch mit Blick auf die hervorgehobene Stellung des Tieres und seines Wohls in Art. 20a GG nicht gerecht. Hervorzuheben ist noch, dass das Bundesverwaltungsgericht in seiner bekannten Entscheidung zur Rechtswidrigkeit der Tötung männlicher Küken aus sogenannten „Legehennenlinien“ vom 13.6.2019 dem Versuch des Vertreters der Brütereibetreiber eine klare Absage erteilte, die die Ausfüllung des ,,vernünftigen Grundes" erst ermöglichende umfassende Interessenabwägung in Frage zu stellen und letztlich jedes auch nur irgendwie geartete „schutzwürdige“ Interesse ausreichen zu lassen, ohne es noch eingehend zum Staatsziel und Verfassungsgut Tierschutz in Relation zu setzen. ${ }^{23}$ Das Gericht führte weiterhin aus: „Dass das Verhalten gegenüber dem Tier nicht willkürlich ist, insbesondere nicht auf zu missbilligenden Motiven beruht, wie etwa der Lust an der Vernichtung oder dem Quälen eines Tieres ... genügt für einen vernünftigen Grund hiernach nicht." ${ }^{24}$ Im Hinblick auf wirtschaftliche Interessen von Tierhaltern an der Tötung von Tieren konkretisierte der Senat weiter: ,Sie sind nicht schon deshalb vernünftig im Sinne von $\$ 1$ Satz 2 TierSch G, weil sie ökonomisch plausibel sind." 25 Die $\int S 1$ S. 2 , 17 Nr. 1, 18 Abs. 1 Nr. 1, 18 Abs. 2 TierSchG enthalten als Verbotsnormen den Grundsatz, Tieren ohne vernünftigen Grund keine ( $\$ 1$ S. 2 TierSchG) bzw. keine erheblichen $(\$ 18$ Abs. 1 Nr. $1, \$ 18$ Abs. 2 TierSchG) Schmerzen, Leiden oder Schäden zufügen oder diese gar töten zu dürfen $(\$ 17 \mathrm{Nr}$. 1 TierSchG). Im Falle eines Verstoßes gegen den Straftatbestand ( $\$ 17$ Nr. 1 TierSchG) oder einen der Ordnungswidrigkeitstatbestände $(\$ 18$ Abs. 1 Nr. $1, \rrbracket 18$ Abs. 2 TierSchG) kann durch den ,vernünftigen Grund“ als Rechtfertigungsgrund allenfalls die Rechtswidrigkeit entfallen. ${ }^{26}$

\section{Die besondere Stellung des Zoos und seine Grundrechts- bzw. Staatszielbindung}

In Deutschland sind viele der ca. 900 unter die weite Zoodefinition des $\int 42$ Abs. 1 BNatSchG fallenden Einrichtungen $^{27}$ zumindest teilweise in öffentlicher Hand. Von den 56 deutschen ${ }^{28}$ im Verband der zoologischen Gärten e. V. (VdZ) vereinigten Zoos, dessen Mitglieder für sich in Anspruch nehmen, ,wissenschaftlich geführt ${ }^{\prime 29} \mathrm{zu}$ sein, sind 37 in öffentlicher Hand, 26 davon zu $100 \%$, weitere drei über $90 \%$, drei über $70 \%$, nur fünf unter $50 \%$. Nur 19 der deutschen VdZ-Mitglieder sind reine Privatunternehmen, erhalten aber dennoch, wie z.B. der Zoo Neumünster $^{30}$, nicht unerhebliche staatliche Zuschüsse. Die Träger agieren in den unterschiedlichsten Rechtsformen wie $z$. B. als gemeinnützige $\mathrm{GmbH}^{31}$, als eingetragener Verein ${ }^{32}$ oder die Zoos sind direkte, unselbstständige Eigenbetriebe der wie z.B. beim Tiergarten Nürnberg ${ }^{33}$ oder dem Frankfurter $\mathrm{Zoo}^{34}$

\subsection{Die Vorgaben zur Klassifizierung einer Einrichtung als Zoo}

Eine dauerhafte Einrichtung, in der lebende Tiere wildlebender Arten gerade zum Zwecke der Zurschaustel-

19) Dazu auch BVerwG, Urt. v. 13.6.2019 - 3 C 28.16 und 3 C 29.16, Rdnr. 20, NuR 2020, 45 m. Anm. Maisack, NuR 2019, 824 ff. und Anm. Arleth, ZUR 2019, 681 ff. - Tötung männlicher Küken.

20) Vgl. Schröter, NuR 2007, 468, 469.

21) So zuletzt BVerwG, Urt. v. 13.6.2019 - 3 C 28.16 und 3 C 29.16, Rdnr. 17; BT-Drs. 16/9742, S. 4; Hirt/Maisack/Moritz, Tierschutzgesetz, 3. Aufl. 2016, \1 Rdnr. 31, 33.

22) OLG Celle, Urt. v. 12.10.1993 - 2 Ss 147/93StA, NuR 1994 515. Ähnlich auch Caspar, NuR 1997, 577.

23) BVerwG, Urt. v. 13.6.2019 - 3 C 28.16 und 3 C 29.16 , Rdnr. 17 ff., 27, dazu Anm. Arleth, ZUR 2019, 681, 686.

24) BVerwG, Urt. v. 13.6.2019 - 3 C 28.16 und 3 C 29.16, Rdnr. 18

25) A.a.O.

26) Vgl. Hirt/Maisack/Moritz, Tierschutzgesetz, 3. Aufl. 2016, \1 Rdnr. 34; Caspar, NuR 1997, 577. A.A. BayObLG, Beschl. v. 21.3. 1977 - RReg. 4 St 44/7, BayObLGSt 1977, 41; Metzger, in: Lorz/Metzger, Tierschutzgesetz, 7. Aufl. 2019, \$17 Rdnr. 2, 34; Dietlein, NStZ 1994, 21, 22.

27) Hierzu zählen auch die zahlreichen Wildgehege und Wildparks, in denen klassischerweise nur einheimische Tiere gehalten werden, sowie Aquarien, Vogelhäuser oder Streichelzoos.

28) Verbands der Zoologischen Gärten (VdZ) e. V.: Zoos im VdZ, Stand 2.3.2021, abrufbar unter https://www.vdz-zoos.org/mitglieder/zoos-im-vdz.

29) Vgl. Leitbild des VdZ e. V., Stand 2.3.2021, abrufbar unter https://www.vdz-zoos.org/verband/leitbild-und-faktenblatt.

30) Holsteinischer Courier: Neumünster: Der Tierpark bekommt 200000 Euro, Stand 2.3.2021, abrufbar unter https://www. shz.de/lokales/holsteinischer-courier/der-tierpark-bekommt200-000-euro-id27416852.html.

31) Wie z.B. der Erlebnis Zoo Hannover, dessen Träger die Zoo Hannover $\mathrm{GmbH}$ ist, vgl. Auszug aus dem Gesellschaftsvertrag der Zoo Hannover gemeinnützige GmbH v. 17.12.2009, Stand 2.3.2021, abrufbar unter https://www.zoo-hannover.de/ de/aktuelles/news/Erlebnis-Zoo-als-gemeinnuetzig-anerkannt.

32) Wie z.B. laut Satzung der Tierpark Neumünster, dessen Träger der Tierparkvereinigung Neumünster e. V. ist, Stand 2.3.2021, abrufbar unter https://tierparkneumuenster.de/de/der-tierpark/ informationen-mitgliedschaft.

33) Vgl. die Angaben laut Impressen, Stand 2.3.2021, abrufbar unter https://tiergarten.nuernberg.de/impressum.html.

34) Vgl. die Angaben laut Impressen, Stand 2.3.2021, abrufbar unter https://www.zoo-frankfurt.de/impressum/. 
lung $^{35}$ während eines Zeitraumes von mindestens sieben Tagen im Jahr gehalten werden, ist nach $₫ 42$ Abs. 1 BNatSchG rechtlich als Zoo zu bezeichnen und muss gewisse Voraussetzungen und Anforderungen erfüllen, damit der Betrieb der Einrichtung aufrechterhalten werden darf, vgl. \42 Abs. 3, 6, 7 BNatSchG. Sie unterliegt deshalb einem Genehmigungsvorbehalt durch die zuständige Behörde, vgl. \42 Abs. 2, 7 BNatSchG. Erstaunlicherweise wird in der Literatur bei der Prüfung der Dauerhaftigkeit teilweise auf den gewerbsmäßigen Betrieb sowie die Erhebung von Eintrittsgeldern als wesentliche Kriterien abgestellt. ${ }^{36}$ Dies ist nicht nur schwer mit der Legaldefinition des $\$ 42$ Abs. 1 BNatSchG vereinbar, da diese eben gerade nicht an die GewerbsmäBigkeit anknüpft, sondern an die bloße Dauerhaftigkeit. Auch die teleologische Auslegung spricht gegen eine solche kommerzialisierende Lesart der „Dauerhaftigkeit“, da $₫ 42$ Abs. 1 S. 1 BNatSchG für die Erfüllung des Zurschaustellungszwecks und damit die Eigenschaft, als Zoo zu gelten, bereits sieben Tage im Jahr ausreichen lässt. Schließlich ist offenkundig, dass die Erhebung von Eintrittsgeldern wegen ihrer regulierenden Auswirkungen auf das Besuchsinteresse in einem Spannungsverhältnis zum gesetzlich vorgesehenen Zurschaustellungszweck steht. Bereits an dieser aus den beschriebenen Gründen kaum vertretbaren Berücksichtigung eines rein monetären Faktors (in diesem Beispiel im Genehmigungsverfahren) lässt sich ablesen, dass wirtschaftlichen Aspekten im Zookontext selbst von Gesetzeskommentatoren ein nicht unerhebliches, bei genauerer Betrachtung aber rechtlich nicht berücksichtigungsfähiges Gewicht beigemessen wird.

Weiter sind Zoos nach $₫ 42$ Abs. 3 BNatSchG, ,so zu errichten und zu betreiben, dass 1. bei der Haltung der Tiere den biologischen und den Erhaltungsbedürfnissen der jeweiligen Art Rechnung getragen wird ... ,und „4. die Vorschriften des Tierund Artenschutzes beachtet werden" ". ${ }^{37}$ Die Einhaltung geltenden Tierschutzrechts ist von den Zoos also als dauerhafte Betreiberpflicht ${ }^{38}$ sicherzustellen und nach $₫ 42$ Abs. 6 S. 1 $\mathrm{BNatSchG}$ behördlich durch, ,regelmäßige Prüfungen und Besichtigungen" zu überwachen; die Auskunfts- und Duldungspflichten des $₫ 52 \mathrm{BNatSchG}$, die insbesondere auch Betretungs- und Akteneinsichtsrechte der Behördenvertreter in den Einrichtungen umfassen, gelten qua des Verweises in $\$ 42$ Abs. 6 S. 2 BNatSchG entsprechend. ${ }^{39}$ Eine solche Überprüfungspflicht liefe ins Leere, wenn die Betreiberpflichten nur singulär bei der erstmaligen Genehmigungserteilung vorliegen müssten. Kommt ein Betreiber Anordnungen der Behörden zur Einhaltung der Voraussetzungen des $\lceil 42$ Abs. 3 BNatSchG und damit auch der Vorschriften über den Tierschutz nicht nach, die auch nachträgliche Anpassungen ,an die Haltung von Tieren in Zoos entsprechend dem Stand der Wissenschaft" umfassen können (vgl. $\$ 42$ Abs. 7 S. 3 BNatSchG), ist der Zoo zu schließen und die Genehmigung zum Betrieb zu widerrufen ( $\$ 42$ Abs. 8 S. 1 BNatSchG, $\$ 49$ Abs. 2 S. 1 Nr. 1 Alt. 1 VwVfG). Dadurch, dass die Einhaltung der Vorschriften des Tierschutzrechts also eine Grundvorrausetzung für die Genehmigungsfähigkeit des Betreibens von Zoos darstellt, beanspruchen die Regelungen des Tierschutzrechts auch hinter Zoomauern dieselbe rechtliche Geltung wie gegenüber anderen Tierhaltern oder der Allgemeinheit. Zoos sind kein tierschutzrechtliches Niemandsland. Im Gegenteil kommt ihnen wegen ihrer Staatsnähe sowohl im Rahmen direkter staatlicher Beteiligungen als auch wegen ihres Bildungsund Forschungsauftrags sowie ihres mehrheitlichen Subventionsbedarfs eine erhebliche gesellschaftliche Vorbildfunktion gerade in einem Bereich wie dem Tierschutz zu, der sogar vom verfassungsgebenden Gesetzgeber als Staatsziel angesehen wird. Bevor die Praktiken der Tiertötungen auf ihre Tierschutzrechtskonformität überprüft werden (4), soll der Frage nachgegangen werden, inwieweit sowohl
Zoos mit staatlicher Beteiligung als auch rein privat getragene Einrichtungen an das besondere Schutzniveau des Art. 20a GG gebunden sind, sodass die Vorgaben des $\$ 42$ Abs. $3 \mathrm{BNatSchG}$, insbesondere die der Nr. 4, die auf die Vorschriften des Tierschutzgesetzes (hier: $\$ 17$ Nr. 1 TierSchG) verweist, in einem tierwohlfreundlichen Lichte auszulegen sind.

\subsection{Staatszielbindung Zoo betreibender Unternehmen mit staatlicher Beteiligung}

Nach der tradierten Grundrechtslehre binden die Grundrechte nicht nur die drei Staatsgewalten (Art. 1 Abs. 3 GG), sondern auch juristische Personen, die vollends durch die öffentliche Hand beherrscht werden. ${ }^{40}$ Diese Grundsätze der Grundrechtsbindung sind auf Staatszielbestimmungen jedenfalls insoweit direkt übertragbar, als ein Rechtsträger zwar privatrechtlich organisiert ist, tatsächlich aber mehrheitlich der öffentlichen Hand zuzurechnen ist. Im Umkehrschluss hierzu sind juristische Personen, die zu keinem Anteil in öffentlicher Hand sind, bis auf die Ausnahmen der Art. 1 Abs. 1 GG sowie Art. 9 Abs. 3 S. 2 GG ${ }^{41}$ grundsätzlich nur indirekt über Generalklauseln und unbestimmte Rechtsbegriffe an Grundrechte (bzw. Staatszielbestimmungen) gebunden..$^{42}$ In Ausnahmefällen können aber auch diese privaten Unternehmen im Rahmen der mittelbaren Drittwirkung ungeachtet ihrer eigenen Grundrechte ähnlich oder gar identisch weit wie der Staat durch die Grundrechte in die Pflicht genommen werden; dies gilt nach den vom Bundesverfassungsgericht in der Entscheidung zum „Bierdosen-Flashmob" in einem rein privaten Einkaufszentrum aufgestellten Grundsätzen ,insbesondere, wenn sie in tatsächlicher Hinsicht in einer vergleichbaren Pflichten-oder Garantenstellung hineinwachsen wie traditionell der Staat" ${ }^{43}$. Nicht vollends geklärt scheint die Frage zu sein, ob Unternehmen, die teilweise in öffentlicher Hand sind, einer direkten Bindung an die Grundrechte unterliegen. Während das BVerfG in der Fraport-Entscheidung eine zumindest 50,01\%-Beteiligung der öffentlichen Hand an dem Unternehmen ausreichen lässt, um eine unmittelbare Grund-

35) Für die Wesentlichkeit dieses Merkmals Gläß, in: Giesberts/ Reinhardt, BeckOK UmweltR, 57. Ed., Stand: 1.1.2021, \42 BNatSchG Rdnr. 2; ähnlich auch Sanden/Gellermann, in: Landmann/Rohmer, UmweltR, Ed. 93., August 2020, \$42 BNatSchG Rdnr. 8; a.A. Stöckel/Müller-Walter, in: Erbs/Kohlhaas, Strafrechtliche Nebengesetze, 233. EL., Stand: Oktober 2020, \42 BNatSchG Rdnr. 2, der ein Gewinnstreben auch als Hauptzweck für zulässig erachtet. Ähnlich auch Schütte/Gerbig, in: Schlacke, GK-BNatSchG, 2. Aufl. 2016, §42 Rdnr. 9.

36) Gläß, in: Giesberts/Reinhardt, BeckOK UmweltR, 57. Ed., Stand: 1.1.2021, \42 BNatSchG Rdnr. 2; Kratsch, in: Schumacher/Fischer-Hüftle, BNatSchG Kommentar, 2. Aufl. 2010, \42 Rdnr. 5; nicht so bei Sanden/Gellermann, in: Landmann/Rohmer, UmweltR, Ed. 93., August 2020, \$42 BNatSchG Rdnr. 8.

37) Hervorhebung durch die Bearbeiter.

38) Gläß, in: Giesberts/Reinhardt, BeckOK UmweltR, 57. Ed., Stand: 1.1.2021, \42 BNatSchG Rdnr. 8; Schütte/Gerbig, in: Schlacke, GK-BNatSchG, 2. Aufl. 2016, §42 Rdnr. 10.

39) Vgl. Söhnlein, in: Frenz/Müggenborg, BNatSchG, 2. Aufl. 2016, \42 Rdnr. 17 ff.; Heugel, in: Lütkes/Ewer, BNatSchG, 2. Aufl. 2018, $\int 42$ Rdnr. 8.

40) Herdegen, in: Maunz/Dürig, GG-Kommentar, 91. EL, Stand: April 2020, Art. 1 III Rdnr. 54; BVerwG, Urt. v. 18.3.19981 D 88/97, NVwZ 1998, 1083, 1084; Jobst, NJW 2020, 11 f.; Gurlit, NZG 2012, 249, 252 .

41) Weiterführend Neuner, NJW 2020, 1851, 1852

42) Dies ist allerdings nicht so zu verstehen, dass sich hieraus eine Grundrechtsbindung für die juristischen Personen, die in privater Hand sind, ergibt, sondern die Grundrechte auf Privatrechtsverhältnisse ausstrahlen, vgl. BVerfG, Urt. v. 15.1.1958 - 1 BvR 400/57, NJW 1958, 257.

43) BVerfG, Beschl. v. 18.7.2015 - 1 BvQ 25/15, Rdnr. 6, NJW 2015,2485 f. 2485 . 
rechtsbindung des Unternehmens zu bejahen ${ }^{44}$, ohne dabei Bezug auf den öffentlichen Aufgabencharakter des Unternehmens zu nehmen, rückt ein Teil der Lehre unter anderem die Frage deutlich stärker in den Fokus, ob das Unternehmen staatliche Aufgaben wahrnimmt. ${ }^{45}$ Überträgt man dies auf die Zoobetreiber, ergibt sich zumindest nach BVerfG-Rechtsprechung auch für solche Zooträger in Privatrechtsform, die zumindest mehrheitlich in öffentlicher Hand sind, eine direkte Bindung an das Staatsziel des Tierschutzes. Dies folgt nicht nur aus dem Umstand der mehrheitlichen Beteiligung durch die öffentliche Hand, sondern auch daraus, dass zoologische Gärten im Sinne der Rechtsprechung des Bundesverfassungsgerichts zur Grundrechtsbindung Privater ${ }^{46}$ im Hinblick auf ihren Bildungs-, Forschungs-, Artenschutz und Tierschutzauftrag , in tatsächlicher Hinsicht in einer vergleichbaren Pflichten-oder Garantenstellung"stehen wie der Staat.

\subsection{Staatszielbindung Zoo betreibender Unternehmen in Privathand}

Auch sofern ein Zoo durch eine juristische Person oder Personengesellschaft des Privatrechts betrieben wird, die nicht mehrheitlich oder überhaupt nicht in öffentlicher Hand ist, besteht eine Bindung an die Staatszielbestimmung des Art. 20a GG. Art. 20a GG strahlt als Staatsziel und verfassungsgesetzgeberische Wertentscheidung auch selbstständig auf die Auslegung und Anwendung der unbestimmten Rechtsbegriffe in $₫ 42$ Abs. 3 Nr. 1 und 2 BNatSchG aus, die unmittelbar an die privaten Zoobetreiber adressiert sind und weitere durch diese zu erfüllende Genehmigungs- und Betriebsvoraussetzungen enthalten (biologische Bedürfnisse der Tiere, ,,art- und tiergerecht“, ,gute veterinärmedizinische Praxis“). Schließlich gilt Art. 20a GG direkt ,nach Maßgabe von Gesetz und Recht" für alle tierschutzrechtlich relevanten veterinärbehördlichen, strafverfolgungsbehördlichen sowie gerichtlichen Entscheidungen und damit auch und gerade im vorliegenden Kontext als wesentlicher Abwägungsbelang bei der Frage der Rechtmäßigkeit von Zootiertötungen im Rahmen des unbestimmten Rechtsbegriffs ,,vernünftiger Grund“ ( $\$ \oint 1$ S. 2, 17 Nr. 1, 18 Abs. 1 Nr. 1, 18 Abs. 2 TierSchG). Die Ausstrahlungswirkung von Art. 20a GG für private Zoobetreiber bestand damit bereits zeitlich vor der BVerfG-Entscheidung zum „Bierdosen-Flashmob“ und unabhängig von den dort aufgestellten Grundsätzen, erfüllt diese neuen Grundsätze aber ebenfalls. Zoologische Gärten stehen im Sinne dieser Rechtsprechung ${ }^{47}$, in tatsächlicher Hinsicht in einer vergleichbaren Pflichten- oder Garantenstellung"wie der Staat: Den Nummern 1 bis 4 sowie 7 lit. b) des $\int 42$ Abs. 3 BNatSchG liegen maßgeblich Tier- und Artenschutzgesichtspunkte als Betreiberpflichten zugrunde, mit denen die Legislative die Tier- und Artenschutzpflichten des Staates aus dem Verfassungsziel des Art. 20a GG einfachgesetzlich ausgestaltet und konkretisiert und ihre Geltung als Genehmigungsvoraussetzung auch für den privaten Zoobetrieb angeordnet hat. ${ }^{48}$ Weiterhin sind Anforderungen der Förderung der Aufklärung der Öffentlichkeit über den Erhalt der biologischen Vielfalt gemäß §42 Abs. 3 Nr. 6 BNatSchG sowie der Beteiligung an der ,Ausbildung in erhaltungsspezifischen Kenntnissen und Fähigkeiten“"gemäß $\int 42$ Abs. 3 Nr. 7 lit. c) BNatSchG dem Bereich des eigenen Bildungsauftrags des Staats aus Art. 7 Abs. 1 GG vergleichbar. ${ }^{49}$ Art. 7 Abs. 1 GG normiert einen eigenständigen und allgemeinen Bildungsauftrag des Staats und beinhaltet nicht nur die Schulaufsicht. ${ }^{50} \mathrm{Zu}$ diesem Auftrag des Staats gehört zweifelsfrei auch die Bildung des Menschen über seine gemeinsame Abstammung mit den anderen Säugetieren einschließlich der von Charles Darwin entwickelten Evolutionslehre ${ }^{51}$, die Stellung des Menschen im Ökosystem Erde, seine Rolle im „Anthropozän“"52, dem nach den immensen menschengemachten Einflüssen auf eben dieses Ökosystem bezeichneten Erdzeitalter, sowie seine Rolle für den Erhalt dieses Systems und der darin lebenden Arten. Staatsaufgabe ist mithin nicht nur die Förderung der Gewinnung von Kenntnissen über das eigene Sein als Mensch, sondern auch das Verstehen der komplexeren Umwelt, in der der Mensch lebt, mit ihren verschiedenen Facetten. ${ }^{53}$ Auch $\$ 42$ Abs. 3 Nr. 6 und $\$ 42$ Abs. 3 Nr. 7 lit. c) BNatSchG sind damit Ausdruck legislativer Delegation öffentlicher Aufgaben auf mitunter rein private Träger. Schließlich steht als einer der drei alternativ als Genehmigungsvoraussetzung zu erfüllenden Tätigkeitsbereiche des $\$ 42$ Abs. 3 Nr. 7 BNatSchG in lit. a) die Beteiligung der Zoos an „Forschungen, die zur Erhaltung der Arten beitragen, einschließlich des Austausches von Informationen über die Arterhaltung“ zur Wahl, einem Bereich der Wissenschaftsförderung also, die der Staat ebenfalls grundsätzlich als eigene Aufgabe wahrnimmt und an dieser Stelle der Wahrnehmung durch Private überträgt. ${ }^{54}$

Die Ausstrahlungswirkung von Art. 20a GG für Zoo betreibende Unternehmen in Privathand hat damit trotz unterschiedlicher rechtsdogmatischer Herleitung im Ergebnis dieselbe Reichweite wie für staatlich getragene Zoos.

\section{Fallgruppen von Zootiertötungen und deren rechtliche Einordnung}

Im Lichte der bisher dargestellten Ergebnisse bleibt zu prüfen, ob sich Tötungen von Zootieren mit den Argumenten der Zoobetreiber rechtfertigen lassen. Nachfolgend liegt der Fokus auf den fünf Fallgruppen der wirtschaftlichen Beweggründe (4.1), der Zootiertötungen im Rahmen zooeigener Zuchtprogramme (4.2), Verfütterungen von Zootieren an andere Zootiere (4.3), und der Euthanasie (4.4). Da häufig Kombinationen dieser und weiterer Aspekte zur Begründung von Zootiertötungen herangezogen werden, wird dieser Problemkreis unter dem eigenen Punkt des Motiv-

44) Vgl. BVerfG, Urt. v. 22.2.2011 - 1 BvR 699/06, Fraport AG, NJW 2011, 1201, 1203 ff.; BVerfG, Beschl. v. 14.3.2006-1 BvR 2087/03, NVzW 2006, 1041, 1041. Einen konkreten Bezug auf die öffentliche Aufgabenwahrnehmung nimmt das BVerfG indes in BVerfG, Beschl. v. 18. 5.2009 - 1 BvR 1731/05, NVwZ 2009, 1282 vor

45) So insb. Möstl, in: Maunz/Dürig, GG-Kommentar Werkstand: 90. EL Februar 2020,

Art. 87e, Rdnr. 100 ff. Kritisch zu diesem Ansatz Gurlit, NZG 2012, 249, 253.

46) BVerfG, Urt. v. 22.2.2011 - 1 BvR 699/06, Fraport AG, NJW 2011, 1201, $1203 \mathrm{ff}$; BVerfG, Beschl. v. 14.3.2006 - 1 BvR 2087/03, NVwZ 2006, 1041, 1041.

47) Vgl. BVerfG, Urt. v. 22.2.2011 - 1 BvR 699/06, Fraport AG, NJW 2011, 1201, 1203 ff.; BVerfG, Beschl. v. 14.3.2006-1 BvR 2087/03, NVwZ 2006, 1041, 1041.

48) Vgl. BT-Drs. 16/12274, S. 69 f., wonach der Gesetzgeber nicht zwischen öffentlichen und privaten Betreibern differenziert.

49) Vgl. zum Bildungsauftrag des Staats BVerfG, Urt. v. 12.6.2018 -2 BvR 1738/12, NJW 2018, 2695, 2705 m. w. N.; Uhle, in: Epping/Hillgruber, Giesberts/Reinhardt, BeckOK Grundgesetz, 45. Ed., Stand: 15.11.2020, Art. 7 Rdnr. $21 \mathrm{ff}$.

50) Vgl. BVerfG, Urt. v. 6.12.1972-1 BvR 230/70 und 95/71, NJW 1973, 133, 134

51) Vgl. zur Evolutionstheorie als vom staatlichen Bildungsauftrag umfassten Teil der Biologie VG Göttingen, Urt. v. 10.6.20094 A 113/07, BeckRS 2009, 34941 unter Verweis auf das Kerncurriculum.

52) Vgl. zur naturwissenschaftlichen Begründung des „Anthropozän“ als eigenem Zeitalter Crutzen, Geology of mankind, Nature 415 (2002), 23; Steffen/Crutzen/McNeill, The Anthropocene: Are Humans Now Overwhelming the Great Forces of Nature?, AMBIO 36 (2007), $614 \mathrm{ff}$.

53) Vgl. z.B. die Vorgaben des Landes Mecklenburg-Vorpommern für ein Lehramtsstudium mit dem Schulfach „Biologie“, welches auch die Zoologie umfasst. Vgl. ferner BVerwG, Urt. v. 18.6.1997 - 6 C 5/96, NVwZ 1998, 853.

54) So hat z.B. der Freistaat Bayern eine Wissenschaftsförderpflicht in seine Verfassung verankert, Art. 140 Abs. 1 BV. 
bündels gesondert betrachtet (4.5). Soweit Zootiertötungen alleine aus Sicherheitsgründen (z.B. Entlaufen eines Tiers), Tierseuchengründen oder zur geplanten Herstellung von Futter für andere Zootiere erfolgen (betrifft v. a. Schweine, Ziegen, Rinder, die zuvor in den Streichelgehegen der Zoos gehalten wurden), richtet sich die Rechtslage nach den speziellen sicherheitsrechtlichen oder tierseuchenrechtlichen Vorschriften bzw. in letzterem Fall direkt nach $\$ 17 \mathrm{Nr} .1$ TierSchG. Auf diese Fallgruppen wird vorliegend aufgrund ihres Ausnahmecharakters bzw. der vergleichsweise unproblematischen Rechtslage nicht eingegangen. Trotz der sinnvollen Systematisierung des juristischen Problemkreises von Zootiertötungen durch Fallgruppenbildung ist wie immer im Umgang mit unbestimmten Rechtsbegriffen auf Rechtsanwendungsebene jeder Fall mit seinen eigenen konkreten Einzelfallumständen gesondert zu prüfen und abzuwägen. Diese Notwendigkeit zeigt eindrücklich bereits der Blick auf ,den“ vermeintlich einheitlich zu beurteilenden „Fall“ Neumünster, der bereits eingangs angesprochen wurde: Die Zooleitung verlautbarte aufgrund von EinnahmeeinbuBen im ersten „Corona-Lockdown“ bereits im April 2020, Notfallschlachtpläne mit Tötungsreihenfolgen erarbeitet $\mathrm{zu}$ haben und im „Worst Case“ bereit zu sein, den gesamten Tierbestand zu töten. Zunächst sollten Individuen getötet werden, um anderen weiterhin Futter in Form der Getöteten anbieten zu können; anschließend müssten aber auch Fischfresser wie Seehunde oder Pinguine, für die der Zoo mangels Einnahmen kein Futter mehr einkaufen könne, getötet werden, statt sie verhungern zu lassen; schließlich sei auch eine Tötung des 3,6 m großen, ca. $700 \mathrm{~kg}$ schweren Eisbären ,Vitus“ nicht ausgeschlossen, der wegen seines Platzbedarfs kaum an eine andere Anlage zu vermitteln sei. ${ }^{55}$

\subsection{Zootiertötungen aus wirtschaftlichen Gründen?}

Wirtschaftliche Aspekte kommen nach mittlerweile durch das Bundesverwaltungsgericht geklärter Rechtslage nicht schon deshalb als ,,vernünftige Gründe“ im Sinne der $\int \mathbb{S} 1$ S. 2, 17 Nr. 1 TierSchG in Betracht, weil sie rein ökonomisch betrachtet plausibel sind. ${ }^{56}$ Im Gegenteil stehen diesen in der Konzeption des deutschen Tierschutzgesetzes mit dem ethisch begründeten Lebensschutzprinzip jedes einzelnen Tieres $(\mathbb{1} 1 \mathrm{~S} .1 \text { TierSchG })^{57}$ und dem in Verfassungsrang erhobenen Staatsziel des Tierschutzes (Art. 20a GG) ${ }^{58}$ besonders starke Abwägungsbelange entgegen. Im angesprochenen Urteil erkannte das BVerwG die wirtschaftlichen Interessen von Tierhaltern an einem möglichst geringen Haltungsaufwand zwar ebenfalls als dem Grunde nach in die Abwägung einzustellenden Belang an, schränkte die Schutzwürdigkeit aber ausdrücklich auf sogenannte „Nutztiere“ im Zusammenhang mit ,unmittelbaren Ernährungs- und vergleichbaren Bedürfnisse[n] der Menschen" ein..$^{59}$

Die Berücksichtigungsfähigkeit wirtschaftlicher Motive bei Zootiertötungen, die klassischerweise nicht als sogenannte ,Nutztiere" angesehen werden und nicht , unmittelbaren Ernährungs- und vergleichbaren Bedürfnisse[n] der Menschen“ im Sinne der Rechtsprechung dienen, sondern in freier $\mathrm{Na}$ tur teils sogar vom Aussterben bedroht sind, ist damit per se zweifelhaft. Hinzu kommt, dass Zoos jedenfalls nach ihrer gesetzlichen Konzeption wegen ihrer besonderen Aufgaben und Verpflichtungen in den Bereichen des Arten- und Tierschutzes sowie der Aufklärung, Bewusstseinsförderung und Bildung über die biologische Vielfalt und die natürlichen Biotope der Arten rechtlich anders als agrarindustrielle Tierhaltungen zu behandeln sind. Zugunsten der Zoobetreiber streiten zwar (zumindest bei privater Trägerschaft und damit Grundrechtsfähigkeit) die Artt. 2 Abs. 1, 12 Abs. 1 und 14 Abs. 1 S. $1 \mathrm{GG}$, die in die Abwägung einzustellen sind. Sie sind allerdings an der Bindung der Zoobetreiber an Art. 20a GG zu messen und mit dieser im Rahmen von \$17 Nr. 1 TierSchG in praktische Konkordanz zu bringen. Ferner ist nach hiesiger Ansicht zu beachten, dass Zoos sich nicht darauf berufen können, dass im Falle unwirtschaftlicher Entscheidungen der Zoo schließen müsse und damit alle dort lebenden Tiere letztlich getötet werden müssten denn selbst Zoos in rein privater Trägerschaft sind in $\$ 42$ Abs. 3 BNatSchG als Stätten mit besonderem legislativem Naturschutz-, Tierschutz- und Bildungsauftrag konzipiert. Wenn der Staat die Erfüllung (mehrerer) eigener Aufgaben (vgl. Artt. 7 Abs. 1, 20a GG) aber in die Hände Privater legt, muss die öffentliche Hand in einer wirtschaftlichen Notsituation, wie sie etwa im Fall pandemiebedingter Einnahmeausfälle vorliegt (aber auch in anderen, weniger extremen Ausnahmefällen) zumindest für die Kosten aufkommen, die zur Erhaltung des Lebens der in Zoogefangenschaft befindlichen und damit menschlichen Sorge ausgelieferten Tiere anfallen. Etwas anderes ergibt sich auch nicht aus den Regelungen der $₫ 42$ Abs. 8 S. 2 und 3 BNatSchG. Danach sind zwar Zootiere auch nach einer behördlichen Zooschließung grundsätzlich auf Kosten des Betreibers tier- und artgerecht weiter zu behandeln und unterzubringen. Für die ultima ratio, die „Beseitigung“ von Tieren im Kontext von ZooschlieBungen, gelten jedoch weiterhin das Artenschutz- und Tierschutzrecht und damit die strengen Abwägungsmaßstäbe des Art. 20a GG im Rahmen des „,vernünftigen Grundes“, in diesem Fall zusätzlich verstärkt durch die einfachgesetzliche Ausformung in $\$ 42$ Abs. 8 S. 3 BNatSchG am Ende, die besagt, dass eine Tiertötung nur in Betracht kommt, wenn es „keine andere zumutbare Alternative für die Unterbringung der Tiere" gibt. Im Umkehrschluss bedeutet dies, dass bloße finanzielle Engpässe, etwa für die Beschaffung von Futtermitteln, veterinärmedizinische Versorgung oder selbst für den Umbau von Zooanlagen zur Anpassung an neue Haltungsrichtlinien nicht geeignet sind, Zootiertötungen $\mathrm{zu}$ rechtfertigen, sondern von der öffentlichen Hand getragen werden müssen, da diese Maßnahmen insgesamt durch (notfalls staatliche) Geldmittel umsetzbar, für sich betrachtet rein wirtschaftlicher und damit im Rahmen des ,,vernünftigen Grundes“ alleine nicht berücksichtigungsfähig sind. ${ }^{60}$

55) Vgl. die Berichte bei Redaktionsnetzwerk Deutschland v. 13. 4.2020, Stand 2.3.2021, abrufbar unter https://www.rnd.de/panorama/ corona-krise-tierpark-fuhrt-liste-von-tieren-die-getotet-werden-mussten-SGLY2BYZRFB4HDSWGQW7G3RN74.html, in der Süddeutschen Zeitung v. 15.4.2020, Stand 2.3.2021, abrufbar unter https://www.sueddeutsche.de/panorama/corona-krisezoo-notschlachtungen-1.4877112, und dem Spiegel v. 15.4.2020, Stand 2.3.2021, abrufbar unter https://www.spiegel.de/panorama/ coronakrise-zoo-in-neumuenster-erstellt-notschlachtplan-fuertiere-a-e045797c-b868-4780-932d-c1c1d2c19105.

56) Dazu auch BVerwG, Urt. v. 13.6.2019-3 C 28.16 und 3 C 29.16, Rdnr. 18 m.w. N.

57) Weiterführend BVerwG, Urt. v. 13.6.2019 - 3 C 28.16 und 3 C 29.16, Rdnr. 16, 19 m.w. N.

58) Weiterführend BVerwG, Urt. v. 13.6.2019 - 3 C 28.16 und 3 C 29.16, Rdnr. 20, m.w. N.

59) BVerwG, Urt. v. 13.6.2019 - 3 C 28.16 und 3 C 29.16, Rdnr. 18

60) Noch weniger kommen Zootiertötungen in Betracht als Voraussetzung zur Durchführung baulicher Luxusrenovierungen, also Umbaumaßnahmen, die weder tierschutz- noch baurechtlich angezeigt sind, sondern z.B. lediglich aus optischen Gründen erfolgen sollen; selbst wenn damit auch modernere Haltungsbedingungen verfolgt würden, ist nicht ersichtlich, weshalb die vorübergehende Verlegung der betroffenen Tiere in andere Gehege, Zoos oder Einrichtungen als milderes Mittel gegenüber der Tötung zur möglichst schnellen Durchführung der Bauarbeiten unzumutbar sein sollte, insbesondere wären bloße wirtschaftliche Belastungen solcher vorübergehender Verlegungen nicht ,,vernünftig“; vgl. dazu die 2020 erfolgten Tötungen von einem Braunbären und drei Wölfen im Kopenhagener Zoo, an deren ehemaligen Gehegestandorten dann nach den Tötungen ein Kinderspielplatz errichtet wurde, Bericht von RTL: Weil modernisiert wird - Kopenhagener Zoo tötet drei Wölfe und einen Bären, Stand 2.3.2021, abrufbar unter https://www.rtl.de/ $\mathrm{cms} /$ zoo-kopenhagen-toetet-drei-woelfe-und-einen-braunbaeren-um-platz-fuer-neues-zu-machen-4638058.html. 
Die primär vom Betreiber zu erfüllenden Pflichten inkl. der Kosten dafür sind also subsidiär durch den Rechtsträger der zuständigen Naturschutzbehörde zu erfüllen. ${ }^{61}$

Deshalb begründen nach hier vertretener Auffassung Zootiertötungen aus wirtschaftlichen Gründen, egal ob als primäres Motiv oder auch nur als untergeordnetes neben anderen, keinen ,vernünftigen Grund“ im Sinne des $\$ 17$ Nr. 1 TierSchG.

\subsection{Zootiertötungen als „notwendiges Übel“ zooeigener Züchtungen?}

Der am häufigsten von den Zoos zur Erklärung der Zootiertötungen angeführte Rahmen ist die Durchführung sogenannter „Erhaltungszuchtprogramme“ als Mittel eines Artenschutzes ex situ, also außerhalb natürlicher Lebensräume, innerhalb geschlossener Zooumgebungen. Bereits die Differenzierung zwischen ex situ und in situ muss zu denken geben. Es stellt sich die Frage, welche Berechtigung ex-situ-Artenschutz vor dem Hintergrund überhaupt haben kann, dass es unmöglich ist, intelligenten Exoten, die in Freiheit Arbeitsteilung betreiben, über menschenähnliches Sozialverhalten verfügen und nicht selten erlerntes Wissen sowie Riten über Generationen weitergeben ${ }^{62}$, auf künstlich angelegten, vergleichsweise winzigen Flächen, in menschlich vorbestimmten Sozialverbänden und mit vorgesetzter Nahrung auch nur annähernd naturgemäße Lebensbedingungen zu bieten wie in situ. Diese tierschutzrechtliche Kritik wird durch die naturschutzrechtliche ergänzt, da der ex-situ-Artenschutz in den Zoos als ein auf Selbsterhaltung angelegter Mikrokosmos praktiziert wird: die Anzahl erfolgreicher Auswilderungsprogramme von in Freiheit ausgestorbenen oder selten gewordenen Tierarten liegt weltweit bis heute in einem niedrigen zweistelligen Bereich. ${ }^{63}$ Zahlreiche der in Zoos gezüchteten Tierarten sehen zwar faszinierend aus und locken Besucher an, fallen aber nach den Kategorien der Roten Liste der Weltnaturschutzunion noch nicht einmal unter den Status ,gefährdet“. ${ }^{64}$ Angesichts von aktuell 14735 gefährdeten Tierarten auf dieser Liste (Tendenz seit Jahren steigend) ${ }^{65}$ ist die Behauptung, Zoos leisteten einen Beitrag im Kampf gegen das Artensterben, der angesichts des Ausmaßes an Biodiversitätsverlust auch nur als annähernd relevant bezeichnet werden könnte, eine Farce. Daran ändert auch die ebenfalls vernachlässigbare Unterstützung einzelner in-situ-Schutzprogramme durch manche Zoos nichts, da sie sich gemessen am Jahresbudget im Promillebereich bewegt. ${ }^{66}$ In diesem Kontext sind die eigentlichen Fallgruppen zuchtbedingter Tötungen zu sehen und auf ihre konkrete juristische Stichhaltigkeit hin zu überprüfen.

\subsubsection{Unerwünschte Tiere}

Diese Fallgruppe bezeichnet Tiere, die wegen ihres Geschlechts (zumeist männliche Tiere) oder ihrer höheren als geplanten Anzahl als Ergebnis zoointerner Züchtungen von vorneherein nicht von den Zoobetreibern erwünscht sind oder infolge ihres höheren Alters jüngeren nachgezüchteten Tieren aufgrund einer angestrebten Verjüngung des Tierbestands weichen sollen. ${ }^{67}$ Flächendeckend ist die a priori abwertende Deklarierung dieser Tiere als ,überschüssig“ oder ,„überzählig“ zu lesen ${ }^{68}$, die bedauerlicherweise bis heute auch in der rechtswissenschaftlichen Literatur soweit ersichtlich völlig kritiklos rezipiert und repetiert wird. ${ }^{69}$ Damit wird nicht nur psychologisch bereits ein im Tierschutzrecht nicht vorgesehener Minderwert und herabgesetzter Schutzstatus impliziert, der den Weg zu einer voreingenommen tötungsfreundlicheren Güterabwägung im Rahmen von $\ 17$ Nr. 1 TierSchG ebnet. Insbesondere verstellt diese despektierliche Rhetorik auch den Blick und erschwert eine unvoreingenommene Bewertung der Tatbestandsseite. Einer solcher bedarf es aber, um kritisch nach den eigentlichen Ursachen angeblich ,zu hoher“ Tierzahlen und der Mög- lichkeit von Präventivmaßnahmen hiergegen zu fragen, die als mildere Mittel in die Güterabwägung einzustellen wären. Die erste dieser Ursachen liegt in den auf Selbsterhaltung von Zootierpopulationen fokussierten Nachzüchtungen mit der Folge, dass Zoos keine Vorkehrungen treffen, um unerwünschte Tiere auf eine Auswilderung vorzubereiten, und überdies in der Regel nicht bereit sind, mit anderen Einrichtungen, die sich hierauf spezialisiert haben oder Tieren wenigstens deutlich größere Flächen bieten können, zusammen zu arbeiten. Der in die Jahre gekommene Löwe oder ein Primat wird dann eher getötet und an andere $\mathrm{Zo-}_{\mathrm{o}}$ otiere verfüttert, statt ihnen einen Lebensabend in einer dafür spezialisierten Einrichtung zu ermöglichen. Die zweite Ursache für vermeintlich ,zu hohe“ Tierzahlen ist der notorische Platzmangel in den künstlich geschaffenen zoologischen Gärten. Die Zoos liegen historisch bedingt zum Teil inmitten von Großstädten oder sind selbst an den Rändern urbaner Räume von engmaschigen Straßennetzen und anderer Bebauung umgeben, die es unmöglich machen, den dort gehaltenen Tierarten ein ihrem natürlichen Lebensraum auch nur annähernd vergleichbares Platzangebot zu schaffen. Die Problematik unerwünschter nachgezüchteter Tiere in Zoos und die Praxis ihrer Tötung ist damit zwar faktisch systemisch, rechtlich jedoch nicht automatisch legal. Kein Zoo ist gezwungen, hunderte oder gar tausende verschiedener Tierarten zu halten und sich dadurch absehbar in eine hausgemachte Platzproblematik zu manövrieren. Selbst innerhalb der kritikwürdigen gegenwärtigen Zoodenkmuster (vgl. 4.2) besteht jedenfalls in der deutlichen Reduzierung gehaltener Arten und dadurch entstehender

61) So auch Schütte/Gerbig, in: Schlacke, GK-BNatSchG, 2. Aufl. 2016, \42 Rdnr. 41.

62) Exemplarisch Safina, Die Intelligenz der Tiere, 2017; Brensing, Die Sprache der Tiere, 2018

63) 13-20 Erfolge laut dem Direktor des Leibniz-Instituts für Zoound Wildtierforschung Hofer, in: Brauchen wir noch Zoos?, Stand 2.3.2021, abrufbar unter https://www.spektrum.de/ news/zuchtprogramme-in-zoos-sollen-aussterbende-arten-retten/1534649; ca. 50 Erfolge laut Bund gegen Missbrauch der Tiere, in: Deutschlandfunk Kultur, Eine Arche hinter Gittern?, Stand 2.3.2021, abrufbar unter https://www.deutschlandfunkkultur.de/ueber-sinn-unsinn-und-zukunft-von-zoos-eine-arche-hinter.976.de.html?dram:article_id $=457901$.

64) Z.B. Primaten wie Schnurrbarttamarin, Blutbrustpavian, Totenkopfäffchen oder andere wie Wombat, Königspinguin oder Tümmler, weiterführend Sommer: Hinter 1000 Stäben - Tiere im Zoo, Stand 2.3.2021, abrufbar unter https://www.swr.de/ swr2/programm/download-swr-14172.pdf, S. 6; nur 20-25\% aller weltweit bedrohten Säugetierarten werden überhaupt in Zoos gehalten und nur $3 \%$ der bedrohten Reptilien, Conde, An Emerging Role of Zoos to Conserve Biodiversity, Science 331 (2011), $1390 \mathrm{f}$.

65) International Union for Conservation of Nature and Natural Resources: Red List of threatened species, Stand 2.3.2021, abrufbar unter https://www.iucnredlist.org/resources/summarystatistics.

66) Sommer: Hinter 1000 Stäben - Tiere im Zoo, Stand 2.3.2021, abrufbar unter https://www.swr.de/swr2/programm/downloadswr-14172.pdf, S. 6.

67) Vgl. zu diesen von den Zoos angeführten Topoi Verband der Zootierärzte (VZT): Töten von Zootieren, Stand 2.3.2021, abrufbar unter https://www.zootieraerzte.de/wp-content/uploads/ 2018/12/Toeten-von-Tieren-VZT-2018.pdf, S. 6 ff.

68) Exemplarisch Wustmans/Encke, ZEE 2019 (Heft 4), 259 f.; Tierärztliche Vereinigung für Tierschutz (TVT), Stellungnahme zur Tötung überzähliger Tiere im Zoo, Stand 2.3. 2021, abrufbar unter https://www.tierschutz-tvt.de/alle-merkblaetter-und-stellungnahmen/?no_cache $=1 \&$ download $=$ TVT-Stellungn. T\% C3\%B6tung_\%C3\%BCberz\%C3\%A4hliger_Tiere__Apr._ 2009_.pdf\&did=177.

69) Vgl. erst jüngst auch in dieser Zeitschrift Metzger, NuR 2020, 821 , aber auch der ansonsten fortschrittliche TierschutzgesetzKommentar von Hirt/Maisack/Moritz, Tierschutzgesetz, 3. Aufl 2016, $\$ 17$ Rdnr. $58 \mathrm{ff}$. 
ausreichender Reserveflächen eine mildere und praktikable Lösung für die Gewährleistung des tierschutzrechtlich gebotenen Lebensschutzes gegenüber der routinemäßigen Tötung bewusst nachgezüchteter Tiere. Bis aktuell große Tierbestände auf natürliche Art und Weise kleiner würden und mehr Platz mit Reserveflächen für weniger Arten vorhanden wäre, könnte übergangsweise auf getrenntgeschlechtliche Gruppen umgestellt und, wo dies nicht möglich ist, Kontrazeptiva oder notfalls Sterilisierungen einzelner Tiere eingesetzt werden. Dass Zoos gerade diese Mittel mit dem Einwand ablehnen, die Fortpflanzung entspreche den Bedürfnissen und dem natürlichen Verhalten der Tiere, verblüfft, werden andere schwerwiegendere Eingriffe wie namentlich die einkalkulierten Tiertötungen aber z.B. auch das Stutzen bzw. Amputieren der Flügel von Vögeln in Zoos mit dem Ziel, deren Entweichen zu verhindern, routinemäßig vorgenommen und nicht in Frage gestellt. ${ }^{70}$ Schließlich wird der Einwand, dass selbst in freier Natur bei weitem nicht alle Tiere an der Fortpflanzung teilnehmen, sondern häufig nur die (stärksten) Leittiere verschwiegen. Die von den Zoos stereotypisch angeführten Aspekte mangelnder Planbarkeit nachgezüchteter Tiere sowie damit verbundener Platzmangel (dazu 4.5) sind gegenüber den dargelegten milderen und gleich geeigneten Mitteln nur vorgeschoben, da sie nicht nach den eigentlichen Ursachen des Problems fragen, sondern diese möglichst kritiklos perpetuieren wollen. Angesichts der gebotenen Gesamtwürdigung aller Umstände im Rahmen des ,vernünftigen Grundes“ sind die Quantität gezeigter Tierarten und Individuen jedoch ebenso wenig geeignet, eine Tiertötung zu begründen, wie der dadurch gesteigerte Erlebnis- und Werbewert der Zoos.

\subsubsection{Unerwünschte genetische Merkmale und Unfruchtbarkeit}

Ein die Fallgruppe unerwünschter genetischer Merkmale eindrücklich beschreibendes Beispiel, welches in drei Instanzen als Verstoß gegen $\$ 17$ Nr. 1 TierSchG beurteilt wurde, ist das der drei gesunden, von der Mutter nach der Geburt angenommenen und daraufhin noch am gleichen Tag (5.5.2008) im Magdeburger Zoo getöteten Tigerbabys. ${ }^{71}$ Die Elterntiere Colina und Taskan waren zuvor vom Zoo gezielt zur Verpaarung zusammengebracht worden, um - als Teil des Europäischen Erhaltungszuchtprogramms (EEP) - reinrassige sibirische Tigernachkommen zu zeugen. Nachdem dies nach zwei Jahren Gewöhnung der Tiere aneinander gelungen und Colina schwanger war, erhielt der Zoo vom EEP-Koordinator die Mitteilung, Taskan sei nicht reinrassig und daher sofort aus dem EEP ausgeschlossen - dies alleine aufgrund eines geringen Anteils Sumatratiger-Bluts und obwohl umstritten war, ob Sumatratiger biologisch überhaupt als eigene Art oder nur als Unterart zu klassifizieren waren. Der Platz für die Unterbringung der Jungtiere wäre für mindestens zwei Jahre im Zoo Magdeburg gewährleistet gewesen, in denen auch nach den Ausführungen des Landgerichts Ausweichplätze in anderen Einrichtungen oder mit Hilfe von Spenden sogar Kapazitätserweiterungen vor Ort hätten realisiert werden müssen. ${ }^{72}$ In einer von den vier Verurteilten vor der Tötung unterzeichneten gemeinsamen Erklärung, überschrieben mit „Entscheidung zur Euthanasie von Zootieren“, erklärten diese unter dem Punkt ,,bedachte Alternativen“ zudem: „Die Jungtiere sind für die Erhaltungszucht zukünftig wertlos. Eine Abgabe in andere Zoos wird höchstwahrscheinlich nicht möglich sein. Der belegte Platz dieser Tiere nimmt nötigen Platz für reinerbige Tiere. Die genetische Variabilität der verbleibenden Sibirischen Tiger und damit auch die Qualität der Erhaltungszucht litten erheblich." "73 Das LG erteilte dem eine klare Absage: „Die Tötung der drei Tigerwelpen diente jeweils - wie die Angeklagten wussten - nicht unmittelbar dem Artenschutz. Zum Zeitpunkt der Tötung waren die Tigerwelpen nicht geschlechtsreif und hätten sich aufgrund der möglichen Haltung im Zoo jedenfalls für die Dauer von zwei Jahren auch nicht mit anderen Tigern paaren können. Zudem wäre eine Sterilisation der drei gezüchteten Tiger - ebenso wie bei „Taskan“ - möglich gewesen Das OLG Naumburg fügte in dritter Instanz hinzu: „Nirgendwo ist vorgesehen, im Zoo geborene Jungtiere, die nicht zur Erhaltung ihrer Art beitragen können, umzubringen. Weder die Richtlinie 1999/22/EG noch das deutsche Recht oder sonstige nationale oder internationale Regeln, auch nicht das Europäische Erhaltungszuchtprogramm für Sibirische Tiger, legitimieren vorbehaltlos das Töten der gesunden Nachkommen erbgenetisch missglückter Verpaarungen (vgl. auch BT-Drs.: 16/9742, S. 3 u. 5). Gemäß \37 Abs. 2 BNatSchG bleiben die Vorschriften des Tierschutzes von denjenigen des Artenschutzes unberührt." ${ }^{\text {"4 }}$ Diese Grundsätze, anhand derer die drei Gerichte die Interessensabwägung im Rahmen der Prüfung des ,,vernünftigen Grunds“"vornahmen, gestalten die entsprechenden Aussagen des Tierschutzberichts des Bundesministeriums für Ernährung und Landwirtschaft von 1999 zum Thema aus. Schon dort wurden vorübergehende Sterilisierungen, zeitweises Aussetzen der Zucht und die Festlegung eines bestimmten Zuchtturnus als Notwendigkeiten der Geburtenkontrolle in Zoos aufgrund fehlender natürlicher Bestandsregulierung genannt. ${ }^{75} \mathrm{Nach}$ denselben Grundsätzen wäre auch eine Tötung des Löwen Subali im Nürnberger Zoo rechtswidrig, selbst wenn ein Fertilitätstest seine Unfruchtbarkeit ergeben sollte. Weder die fehlende Bereitschaft, weiterhin das Geld und den Platz für die Haltung des Tieres aufzubringen, noch die generelle Ablehnung der bereits organisierten und finanzierten Uberführung Subalis in die südafrikanische Auffangstation wären rechtlich akzeptabel, da sie mildere, realisierbare Mittel gegenüber der Tötung darstellen.

\subsection{Tötungen von Zootieren zur Verfütterung an andere Zootiere?}

Von den zuvor genannten Tötungsgründen zu unterscheiden ist die Tötung von Zootieren, um als Futter für andere im Zoo lebende Tiere verwendet zu werden. Nicht erörtert wird die von vorneherein geplante Aufzucht von Tieren im Zoo zum Zwecke der späteren Verfütterung, die quantitativ einen nicht unerheblichen Teil der Zootiertötungen ausmacht ${ }^{76}$ und, wenn sie im Hinblick auf genau bestimmte $\mathrm{zu}$ fütternde Tiere dokumentiert ist ${ }^{77}$,

70) Die Bundesregierung hält das routinemäßige Flugunfähigmachen von Vögeln in Zoos mangels ,tierärztlicher Indikation im Einzelfall“" (vgl. $\$ 6$ Abs. 1 S. 2 Nr. 1 lit. a) TierSchG) für rechtswidrig, vgl. Antwort auf eine Kleine Anfrage in BTDrs. 18/3792, S. 4; weiterführend dazu auch in dieser Zeitschrift Maisack/Schmidt, NuR 2017, 734 ff.

71) AG Magdeburg, Urt. v. 17.6.2010 - 14 Ds 181 Js 17116/08, BeckRS BeckRS 2010, 17656; LG Magdeburg, Urt. v. 6.12.2010 - 26 Ns 120/10, BeckRS 2011, 1796; OLG Naumburg, Beschl. v. 28.6.2011 - 2 Ss 82/11, BeckRS 2011, 25165; ausführlich auch Hirt/Maisack/Moritz, Tierschutzgesetz, 3. Aufl. 2016, \$17 Rdnr. 58

72) LG Magdeburg, Urt. v. 6.12 .2010 - 26 Ns 120/10, BeckRS 2011, 1796, unter V.

73) LG Magdeburg, Urt. v. 6.12.2010 - 26 Ns 120/10, BeckRS 2011, 1796, unter III. 3.

74) OLG Naumburg, Beschl. v. 28.6.2011 - 2 Ss 82/11, BeckRS 2011, 25165, unter 2. a)

75) BR-Drs. 181/99, S. 38f.

76) Jährlich 30000-40000 in deutschen Zoos laut Dollinger, Nachhaltige Zucht im Zoo - ein Zukunftsprojekt?, in: Tierschutz in Zoo und Zirkus, 2014, S. 74.

77) Zweifelhaft im Fall der StA Magdeburg, 178 Js 25233/11 (= GenStA Naumburg, 111 Zs 1097/12), in dem 27 Zebrafinken im Magdeburger Zoo im Jahr 2011 nach ihrer Tötung erst Monate lang eingefroren und nicht verfüttert wurden und ihre Voliere für den Aufbau einer neuen Zucht von Goldamadinen benutzt wurde, vgl. Volksstimme: Neue Ermittlungen gegen den Zoodirektor. Tiertötungen am Zoo künftig vermeiden, Stand 2.3.2021, abrufbar unter https://www.volksstimme.de/ nachrichten/magdeburg/428030_Tiertoetungen-am-Zoo-kuenftig-vermeiden.html. 
von einem ,,vernünftigen Grund“ im Sinne des TierSchG gedeckt ist. Vorliegend soll es ausschließlich um Tötungen von Zootieren zum Verfüttern gehen, die als (zum Teil auch artenschutzrechtlich besonders geschützte) Nachzüchtungen in Erhaltungszuchtprogrammen erst im Laufe ihres Lebens unerwünscht werden, weil sich z. B. herausstellt, dass sie unfruchtbar sind, alt, krank, männlich, sozial auffällig oder schlicht zu viele für die Zookapazitäten. Die Tötung eines Zootieres zum Zweck des Verfütterns dürfte auch mit Blick auf diese Anlässe nach geltendem Recht nur ultima ratio nach einer umfassenden Gesamtabwägung sein. Sie kommt dann nicht in Betracht, wenn ein schmerz- und leidfreies Leben durch veterinärmedizinische Behandlung weiterhin gewährleistet werden kann oder Zoos lediglich aus den oben beschriebenen hausgemachten Gründen der Überfüllung nicht bereit sind, weiterhin Kost und Logis für ein Tier bereitzustellen. Eine Tötung aus Platzgründen mit anschließender ,Verwertung" als Futter wäre Rechtsmissbrauch. Wer eine Situation, die absehbar zur Erfüllung eines Verbotstatbestands wie einer Tiertötung führt, selbst sehenden Auges herbeiführt, wie dies bei Zoos, die trotz Platzmangels ohne Gegenmaßnahmen weiterzüchten, der Fall ist, kann hieraus keine Rechte (namentlich zur Tiertötung) herleiten (venire contra factum proprium).$^{78}$

Zur Wahrung des Tierwohls ist zu verlangen, dass auch eine Entscheidung über eine Tötung zur anschließenden Verfütterung immer für den konkreten Einzelfall vorgenommen und abgewogen werden muss; dafür muss eine tiermedizinische Indikation dokumentiert sein, also z.B. eine unheilbare, mit Leiden verbundene Krankheit des Tieres. Zudem muss ein diese Entscheidung stützendes Votum der Tierschutzkommission vorliegen ${ }^{79}$, in der nach dem Rechtsgedanken des $\$ 16$ a Abs. 1 S. 2 Nr. 2 HS 3 TierSchG auch mindestens ein amtlicher Veterinär vertreten sein muss. Keine Unterscheidung ist auch hinsichtlich des Alters des Tieres zu machen. Nur im Rahmen dieser engen Grenzen sind Tötungen von Zootieren aus Erhaltungszuchtprogrammen zum Zweck des Verfütterns an andere Zootiere zulässig.

\subsection{Euthanasierung von Zootieren}

Eine Tötung von Tieren kann im Übrigen - auch unter Beachtung der zuvor dargestellten Grundsätze und Besonderheiten der Fallgruppen - jedenfalls nur dann in Betracht kommen, wenn dem Tier durch die Tötung weiteres Leid sowie Schmerzen erspart bleiben. Voraussetzung ist allerdings, dass die Schmerzen und das Leid weder mit Medikamenten gelindert noch durch eine Therapie geheilt werden können. Mit Blick auf die Irrelevanz der Kostenfrage (vgl. 4.1) darf eine Euthanasierung des Tieres nicht in Betracht gezogen werden, wenn die Behandlung oder gar Heilung lediglich wirtschaftlich nachteilig, aber medizinisch möglich ist. ${ }^{80}$ Dies gilt insbesondere auch dann, wenn das Tier nur vorübergehend erkrankt ist und eine Genesung absehbar ist. Problematisch sind die Fälle, die dazwischen einzuordnen sind, insbesondere dann, wenn eine Heilung nicht möglich ist, aber eine dauerhafte Medikamentierung bzw. Therapie das Leiden und die Schmerzen nehmen kann. Die Euthanasierung kann auch in diesem Falle nur ultima ratio sein. Eine Tötung ist demnach lediglich dann gerechtfertigt, wenn das Tier auch trotz Therapie und Medikamentierung nicht weitgehend art- und verhaltensgerecht leben kann und typisches Artverhalten nicht mehr vorliegt (jeweils gemessen an den ohnehin dürftigen Gefangenschaftsumständen eines Zoos und dem Vergleichszustand vor der Erkrankung) oder eine Leidlinderung bzw. Heilung gar nicht möglich ist. Die Tötung zur Leidlinderung ist in diesen Fällen auch dann gerechtfertigt, wenn das Tier nicht als Nahrungsmittel für andere Zootiere verwendet werden kann.

\subsection{Motivbündel}

Der in der Praxis abgesehen von den hier nicht näher behandelten zum späteren Verfüttern herangezüchteten Tieren am häufigsten anzutreffende Fall ist der des Motivbündels. Eine Tötung wird hierbei nicht nur aus einem einzelnen Beweggrund heraus durchgeführt. Es hat sich gezeigt, dass hierbei in fast allen Fällen der Platzmangel in den Zoos eine zentrale Rolle spielt, während die hinzutretenden Umstände sowohl in ihrer Art als auch in ihrem Gewicht variieren. Egal ob unerwünschte genetische Merkmale für die weitere Zucht, physische Imperfektionen und Krankheiten, hohes Alter im Vergleich zu jüngeren Nachzuchten, Verhaltensauffälligkeiten und daraus resultierende potentielle Gefahren durch Auseinandersetzungen unter Tieren, wirtschaftliche Probleme oder zahlenmäßig unkontrolliertes Nachzüchten (ohne Auswilderungen dabei auch nur ansatzweise zu verfolgen): Deutsche und auch internationale Zoos beteuern regelmäßig und orchestrieren das Töten von Tieren in Zoos sei unvermeidbar, da die geringen Platzkapazitäten für die Nachzuchten der gehaltenen Tierarten und Populationen benötigt würden.

Auch im Magdeburger Tigerprozess führte der Zoo den Platzmangel als weiteren Tötungsgrund neben der Genetik an. In den Tatsachenfeststellungen des LG Magdeburg heißt es dazu: „Vielmehr sahen die Angeklagten die zu erwartenden nicht reinerbigen Jungtiere als für die Erhaltungszucht wertlos an und zogen deshalb eine Tötung der Tigerwelpen gleich nach deren Geburt gegenüber einem Versuch vor, diese für den Zeitraum von jedenfalls zwei Jahren noch im Zoo Magdeburg zu halten und in dieser Zeit Anstrengungen für eine weitere Haltung dieser Tiger oder Abgabe an eine geeignete Einrichtung vorzunehmen. Der Bau weiterer Gehege im Zoo ... hätte voraussichtlich Kosten von mehreren $100000,-€$, möglicherweise auch von mehr als $1000000,-€$, verursacht. Über eine derartig hohe Summe verfügte der Zoo ... jedoch nicht. Die Bauzeit hätte mehr als zwei Jahre, eventuell vier bis fünf Jahre, betragen." $" 1$

Es wurde bereits erläutert (4.2), dass die Tötung von Zootieren als Verstoß gegen das Verbot des venire contra factum proprium rechtswidrig ist, wenn der tatsächlich eingetretene Platzmangel aufgrund organisatorischer Mängel wie insbesondere der Haltung und Präsentation zu vieler unterschiedlicher Tierarten sowie (zumindest zeitweiser, da für den Abbau zu hoher Tierzahlen erforderlicher) unterlassener Verhütungsmittelgabe oder getrenntgeschlechtlicher Haltung wissentlich herbeigeführt wurde. Fraglich ist, wie mit Motivbündeln zu verfahren ist, insbesondere wenn sich darunter gleichzeitig sowohl rechtlich unzulässige als auch zulässige Motive befinden. Zum einen könnte für die Rechtfertigung die Gesamtheit aller Beweggründe in einer wertenden Betrachtung heranzuziehen sein; zum anderen könnte der Beweggrund für die Rechtfertigung maßgeblich sein, der im Schwerpunkt das tragende Element der

78) So auch Hirt/Maisack/Moritz, Tierschutzgesetz, 3. Aufl. 2016 , \$17 Rdnr. 13; AG Magdeburg, Urt. v. 17.6.2010 - 14 Ds 181 Js 17116/08, unter Punkt III. 2, BeckRS 2010, 17656: „In Zoos hat die Zuchtplanung so zu erfolgen, dass die Unterbringung der Nachkommen in jedem Fall gesichert ist." Der Rechtsgedanke von venire contra factum proprium als „Verwirkung“ gilt in der gesamten Rechtsordnung, vgl. BVerfG, Beschl. v. 5.7.1972 - 2 BvL 6/66, 28/69, 3, 11, 12/70, NJW 1972, 1851, 1853 - Anwendung im öffentlichen Recht.

79) Tierärztliche Vereinigung für Tierschutz (TVT), Stellungnahme zur Tötung überzähliger Tiere im Zoo, Stand 2.3.2021, abrufbar unter https://www.tierschutz-tvt.de/alle-merkblaetter-undstellungnahmen/?no_cache $=1 \&$ download $=$ TVT-Stellungn. T\%C3\%B6tung_\%C3\%BCberz\%C3\%A4hliger_Tiere__Apr._ 2009_.pdf\&did=177. S. 4.

80) So auch Hirt/Maisack/Moritz, Tierschutzgesetz, 3. Aufl. 2016, \$17 Rdnr. 84

81) LG Magdeburg, Urt. v. 6.12.2010 - 26 Ns 120/10, BeckRS 2011, 1796, unter III. 
Entscheidung darstellt, also bei objektiver Betrachtung eines Dritten als Hauptbegründung bzw. Hauptzweck einzuordnen ist. ${ }^{82}$ Gegen ersteres Vorgehen sprechen zwei gewichtige Gründe: Hierdurch könnten Beweggründe, die keinesfalls $\mathrm{zu}$ rechtfertigen sind, de facto rechtfertigbar werden, sofern neben einen solchen Beweggrund ein oder mehrere andere treten, die eine Rechtfertigung begründen können. ${ }^{83}$ Zudem würde ein solches Vorgehen aufgrund der denkbaren Vielzahl an Nebenzwecken für tierbelastende Handlungen die vom Gesetz vorgesehene Verhältnismäßigkeitsprüfung konterkarieren. ${ }^{84}$ Weiter widerspräche es in der vorzunehmenden Gesamtabwägung evident dem Individualtierschutz sowie dem Wertungsgehalt des Art. 20a GG, da Zoos ansonsten einen Freischein zum beliebigen „Auswechseln“ von Tieren erhielten. Schließlich muss eine solche Praxis aus generalpräventiven Erwägungen heraus unterbunden werden: Die im Ergebnis rechtlich gebilligte Hinnahme einer Legalisierung des eigentlich Illegalen durch eine beliebige und nicht kontrollierbare Verknüpfung desselben mit dem Legalen würde das Vertrauen in die Durchsetzungskraft des Rechts sowie die erwünschte Abschreckung vor der Begehung ähnlich gelagerter Taten ad absurdum führen. ${ }^{85}$ Ferner zeigt ein Blick in andere Rechtsbereiche, dass auch bei der Auslegung und Rechtsanwendung in anderen Gebieten als dem Tierschutzrecht bei Vorliegen mehrerer Begründungen bzw. Zwecke regelmäßig eine Schwerpunktbetrachtung aus Sicht eines objektiven Dritten erfolgt. ${ }^{86}$ Anhand der für solche Schwerpunktbetrachtungen entwickelten Grundsätze ist auch der ,vernünftige Grund“ im Rahmen von $\int 17$ Nr. 1 TierSchG zu bestimmen. So ist bei lebensnaher Betrachtung z. B. beim sog. Königsfischen (Angelwettbewerb) ein nur vorgeschobener oder allenfalls sekundärer Aspekt der Nahrungserwerb des Anglers, was zumeist schon daran zu erkennen ist, dass die Fische bereits gefangen sind und dann alleine für den Wettkampf in einen Teich oder Bachlauf gesetzt werden. Der entscheidende Beweggrund für die Tötung des Fisches ist in diesem Falle die Teilnahme an einem Wettbewerb, zumeist in Verbindung mit Preisen für die Angler der schwersten bzw. meisten Fische. In einer solchen Situation ist für die Tötung daher lediglich das private Vergnügen der tragende Beweggrund und deshalb die Grundlage zur Prüfung der Rechtfertigung. Für das Beispiel bedeutet dies, dass das Töten von Fischen im Rahmen von Wettbewerben nicht gerechtfertigt ist. ${ }^{87}$ Aus diesen Gründen ist auch bei Zootiertötungen eine Schwerpunktbetrachtung im Rahmen des ,vernünftigen Grundes“ unumgänglich und die von Metzger ${ }^{88}$ vertretene Auffassung, wonach auch nur ein legales Motiv andere illegale ,heile“, klar abzulehnen.

\section{Fazit}

Ein Großteil der Menschen assoziiert mit Tierschutz im Allgemeinen das örtliche Tierheim, welches sich um ausgesetzte Tiere kümmert und diese vermittelt sowie immer mehr auch Recherchen mit erschreckenden Bildern aus der massenhaften Haltung und Tötung sogenannter „Nutztiere" und aus Tierversuchslaboren. Anhand der Ausführungen konnte aufgezeigt werden, dass insbesondere auch solche Tiere in Zoos eines ernst zu nehmenden rechtlichen Schutzes bedürfen, die vermeintlich gut versorgt werden und die sich in Obhut von Menschen befinden, die a prima vista behaupten, stets im besten Interesse der Tiere und der Natur zu handeln. Der Beitrag und insbesondere die diskutierten Fallbeispiele, welche tief in das Selbstverständnis der Zoos und ihre Beziehung zu den dort lebenden Tieren blicken lassen, konnten darlegen, dass Veterinärbehörden und Staatsanwaltschaften Zoos durch mangelnde Kontrollen bzw. unkritische Übernahme von Zoojargon in Einstellungsbescheide unter dem Deckmantel von (vermeintlichem) Naturschutz, Bildung und Wissenschaft keine faktischen Unbedenklichkeitsatteste ausstellen dürfen, sondern im Gegenteil tierschutzrechtlich besonders vorgewarnt sein müssen. Bei dogmatisch korrekter Verhältnismäßigkeitsprüfung im Rahmen des ,,vernünftigen Grundes" (\$S 1 S. 2, 17 Nr. 1 TierSchG) unter Berücksichtigung des Art. 20a GG, an den im Ergebnis auch rein private Zoos gebunden sind (3.3), und unter Berücksichtigung des im Falle behaupteten Platzmangels oder wirtschaftlicher Schwierigkeiten standardmäßig erfüllten Verstoßes gegen das Verbot des ,venire contra factum proprium“ sind daher Tötungen von im Zoo lebenden Tieren grundsätzlich rechtswidrig und strafbar. Das bloße Rekurrieren auf abstrakte Schemata, wie etwa in der schriftlichen Erklärung der vier Mittäter zur Tötung der Magdeburger Tigerbabys nachzulesen, kann diesen Grundsätzen nicht gerecht werden. Tötungen von in Zoos lebenden Tieren können daher abgesehen von tierseuchen- und sicherheitsrechtlichen Notfällen im Ergebnis nur dann nicht rechtswidrig sein, wenn es sich um nachweislich ausschließlich zur Fütterung gezüchtete Tiere oder um amtstierärztlich bestätigt unheilbar kranke und leidende Tiere handelt.

82) So für $\$ 17$ Nr. 1 TierSchG ausdrücklich KG Berlin, Beschl. v. 24.7.2009 - (4) 1 Ss 235/09, NStZ 2010, 175; LG Magdeburg, Urt. v. 6.12.2010 - 26 Ns 120/10, dort unter V., BeckRS 2011, 1796; OVG Koblenz, Urt. v. 28.5.1998 - 12 A 10020/96, Rdnr. 39, BeckRS 1998, 22107; Hirt/Maisack/Moritz, Tierschutzgesetz, 3. Aufl. 2016, \$1 Rdnr. 33; Ort, NuR 2010, 853, 855 und 858.

83) Noch umfangreicher sogar Metzger, in: Erbs/Kohlhaas, Strafrechtliche Nebengesetze, 233. EL., Stand: Oktober 2020, $\$ 17$ TierSchG Rdnr. 16 (es reiche bei einem Motivbündel aus, wenn nur ein Motiv von vielen die Rechtswidrigkeit entfallen lasse).

84) Hirt/Maisack/Moritz, Tierschutzgesetz, 3. Aufl. 2016, \$1 Rdnr. 43

85) Fischer, Strafgesetzbuch, 68. Aufl. 2021, \$46 Rdnr. 2; BVerfG, Urt. v. 21.6.1977 - 1 BvL 14/76, Rdnr. 215 ff., NJW 1977, 1525

86) Vergleichbar ist z.B. die Festlegung der Parameter für die Angebotswertung im Vergaberecht, die anhand einer Schwerpunktbetrachtung vorgenommen wird, vgl. Delcuvé, JuS 2020, 1128 1131 oder einer Schwerpunktbetrachtung im Insolvenzrecht, vgl. BGH, Urt. v. 5. 4. 1978 - VIII ZR 42/77, NJW 1978, 1383, 1384

87) Zutreffend daher die Einordnung bei Pfohl, Joecks/Miebach, Münchener-Kommentar-StGB，3. Aufl. 2017, \$17 TierSchG Rdnr. 59, 61; Drosse, NStZ 1990, 72; StA Hanau 3.5.1991 - 3 Js 13 610/90, NuR 1991, 501; a. A. Jendrusch/Niehaus, NuR 2007, 740.

88) In: Erbs/Kohlhaas, Strafrechtliche Nebengesetze, 233. EL., Stand: Oktober 2020, $\$ 17$ TierSchG Rdnr. 16. 